\title{
The Observation of Self-Channeling of a Relativistically-Intense
}

Laser Pulse in an Underdense Plasma

C. E. Clayton, P. Muggli, D. Gordon, K. C. Tzeng, W. B. Mori, C. Joshi, UCLA;

A. Modena, Z. Najmudin, A. E. Dangor, Imperial College; V. Malka, Ecole Polytechnique; and D. Neely, Rutherford Appleton Laboratory

\section{Abstract}

Four spatial/spectral imaging diagnostics show relativistic self-channeling of a $25 \mathrm{TW}, 1 \mathrm{ps}$ laser pulse limited only by the length of the gas-jet target. Collective scattering of a probe beam provides a spectrally-resolved image of large-amplitude plasma waves indicating an intensity $>10^{18} \mathrm{~W} / \mathrm{cm}^{2}$ at $4 \mathrm{~mm}$ from the laser focus.

The ability to experimentally extend the propagation distance of a very intense laser pulse well beyond a Rayleigh length $x_{R}$ in an underdense plasma is crucial for such applications as x-ray lasers [1], the "fast ignitor" fusion concept [2], and laser-plasma acceleration schemes [3]. The two main approaches to this are injection of the intense pulse into a preformed "plasma fiber" [1,4,5] which, although showing promise, has not been developed to the extent that the above mentioned applications require. The other approach is to allow the intense pulse itself to create its own optical fiber by transversely modifying the refractive index of the plasma [6]. This occurs when the laser power $P$ exceed the critical power $P_{c r}$ for relativistic self-guiding by a factor of 1-3 or $P>1-3 \times P_{c r}$ where $P_{c r}$ $\approx 17\left(n_{\mathrm{cr}} / \mathrm{n}_{\mathrm{e}}\right) \mathrm{GW}$ and $\mathrm{n}_{\mathrm{cr}}$ and $\mathrm{n}_{\mathrm{e}}$ are the critical density (laser frequency $\omega_{\mathrm{o}}=$ plasma frequency $\left.\omega_{\mathrm{p}}\right)$ and the density of the plasma, respectively. The radial modification of the refractive index has two contributions; (1) the relativistic correction to the mass of the plasma electrons due to the quiver motion in the laser field is generally larger on axis than off, and (2) the radial ponderomotive force expels plasma electrons from the axis. These two effects always act

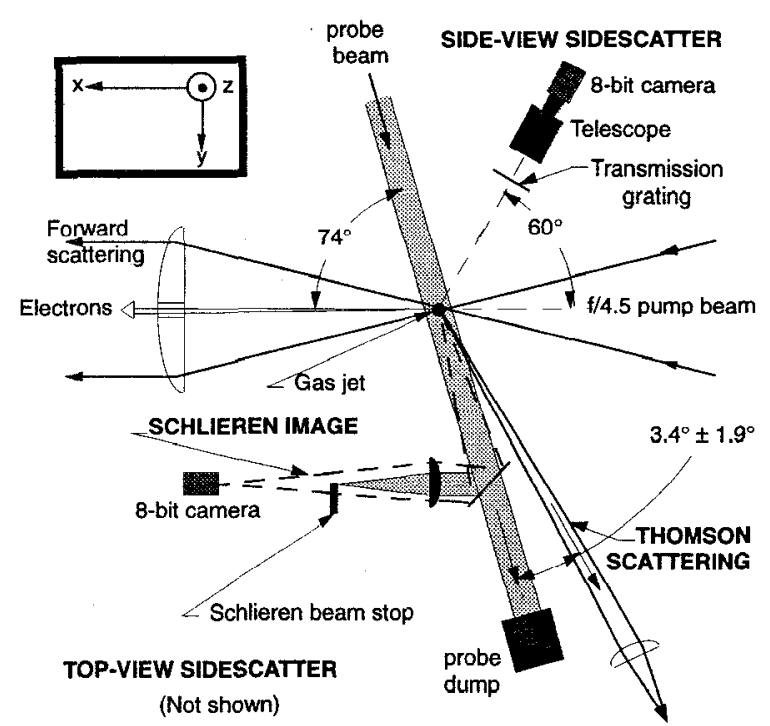

Figure 1. Experimental setup with the four imaging diagnostics labeled in bold type. Forward scattered light (pump and a cascade of Stokes and anti-Stokes) are measured on each shot. Energetic electrons are sent through an $f / 60$ hole in the forward-scattered light collection optics and into a multichannel imaging electron spectrometer.

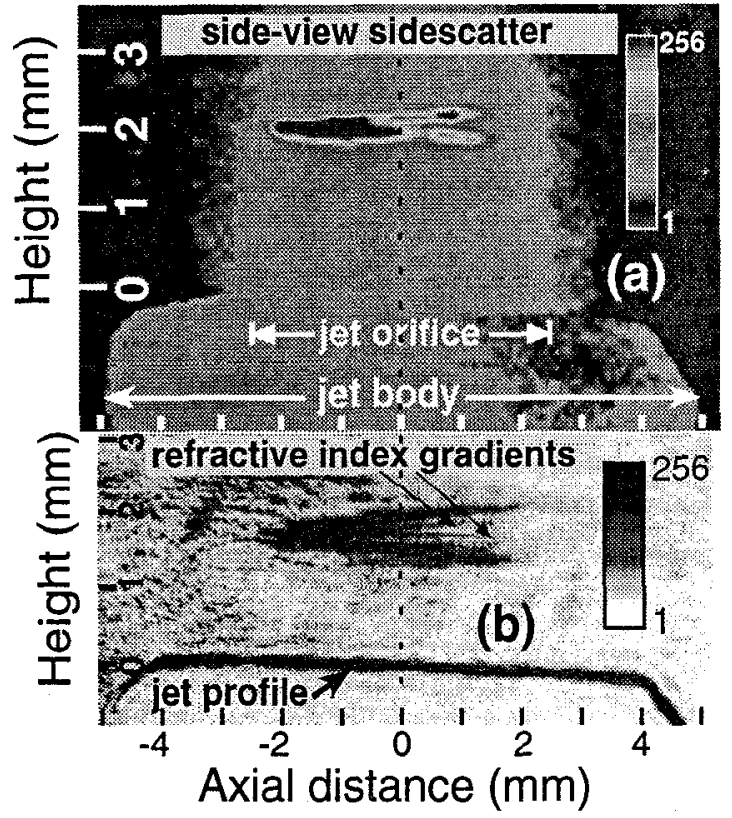

Figure 2. (a) Side-view sidescatter of light near 1 $\mu \mathrm{m}$. (b) Schleiren image taken on the same shot. The fine "whiskers" (labeled with arrows) show the gradients associated with the channel while the course, outer gradients are due to the bulk plasma formed with the $f / 4.5$ focused laser beam (color figure).

together to produce a higher on-axis index which guides the laser pulse into fresh plasma further downstream. Evidence of relativistic guiding has been seen in images of either plasma recombination light or sidescattered pump light [7] or its second harmonic [8,9]. Interferometry has also been employed to visualize these channels at later times $[9,10]$.

In this work [11], four simultaneous, single-shot imaging diagnostics were on-line as shown in Fig. 1. The standard sidescatter diagnostic has been extended by viewing the light through a transmission grating revealing in the 
first diffracted order a spectrally-resolved image of the channel(s). Also, precise spatial correlation of sidescatter with Schleiren photography confirms that radial refractive-index gradients are indeed guiding the light, as shown in Fig. 2. Finally, collective Thomson scattering off electron plasma waves (EPW) within the channel show EPW's existing at the far edge of the gas jet target, $12 \mathrm{x}_{\mathrm{R}}$ from the laser focus at the front edge of the jet plume. This is shown in Fig. 3 along with sidescatter from the same shot. In Figs. 2 and 3, the laser is focused at $x=-2 \mathrm{~mm}$. Correlations between bright sidescatter and large EPW's can be seen suggesting that the channel did not become completely evacuated of electrons by the ponderomotive force, i.e. "cavitation" [12] did not occur. This is perhaps due to collective phenomena within the channel such as Raman-heating of the background plasma which would increase the thermal pressure thus resisting electron blowout [13]. Indeed, single-shot electron spectra reveal spectra extending out to $40-100 \mathrm{MeV}[14,15]$ highlighting the importance of collective laser-plasma instabilities in the channel. Estimates of the normalized EPW amplitude $\delta n / n_{e}$ near the exit of the channel of about $40 \%$ (based on harmonic content of the wave) imply that the exiting laser intensity was still $>10^{18} \mathrm{~W} / \mathrm{cm}^{2}$. It should be noted that on some shots, the measured EPW is only about $600 \mu \mathrm{m}$ long, yet the electron spectrum extended to $100 \mathrm{MeV}$ [15]. This is in excess of the linear dephasing energy $[3,15]$. Also, longer channels tended to have a lower maximum electron energy which could be attributed to phase velocity non-uniformities which can de-trap electrons or enhance radial losses.
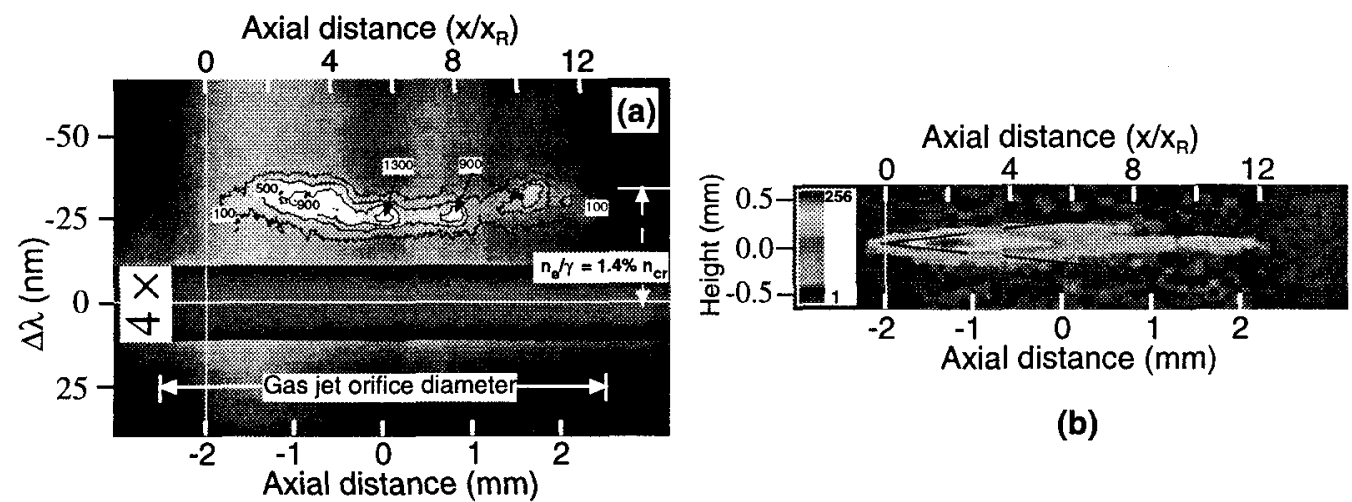

(b)

Figure 3. (a) Frequency-resolved image of EPW amplitude along the laser propagation axis. Contours are of constant scattered probe energy and are artificially suppressed at the edges relative to $\mathrm{x}=0$ due to the temporal profile of the probe pulse [11]. Spatially-modulated Bremsstrahlung continuum is also apparent. (b) Side-view sidescatter near $1 \mu \mathrm{m}$ for the same shot (color).

\section{Acknowledgments}

The authors would like to thank C. Danson at the Vulcan Laser Center and his staff for excellent user support. This work was supported by EPSRC, the EU, the Laboratoire Europeen Associe' LULI/DRAL and the U. S. Department of Energy.

\section{References}

1. N. H Burnett and G. D. Enright, IEEE J. Quant. Electron. 26, 1797 (1990).

2. M. Tabak et al., Phys. Plasmas 1,1626 (1994).

3. E. Esarey et al., IEEE Trans. Plasma Sci 24, 252 (1996).

4. C. G. Durfee III and H. M. Milchberg, Phys. Rev. Lett. 71, 2409 (1993).

5. W. P. Leemans et al., IEEE Trans. Plasma Science 24, 331 (1996).

6. C. Max et al., Phys. Rev. Lett. 33, 209 (1974).

7. A. B. Borisov et al., Phys. Rev. Lett. 68, 2309 (1992); P. Monot et al., Phys. Rev. Lett. 74, 2953 (1995);

K. Krushelnick et al., Phys. Rev. Lett. 78,4047 (1997); R. Wagner et al., Phys. Rev. Lett. 78, 3125 (1997).

8. V. Malka et al., Phys. Plasmas 4, 1127 (1997).

9. M. Borghesi et al., Phys. Rev. Lett. 78, 879 (1997).

10. P. E. Young and P. R. Bolton, Phys. Rev. Lett. 77, 4556 (1996).

11. C. E. Clayton et al., submitted to Phys. Rev. Lett., June 1997.

12. G.-Z. Sun et al., Phys. Fluids 30,526 (1987).

13. K.-C. Tzeng et al., to be submitted.

14. A. Modena et al., Nature 377,606 (1995).

15. D. Gordon et al., submitted to Phys. Rev. Lett., April 1997. 